\title{
Small-Scale Structure Deduced from X- and $\gamma$-ray Timing Measurements
}

\author{
M. Coleman Miller \\ University of Maryland, Department of Astronomy, College Park, MD, \\ $U S A$
}

\begin{abstract}
X-ray timing observations of neutron stars and black holes are among the few available probes of ultrastrong magnetic fields, strong gravity, high densities, and the propagation of thermonuclear burning. Here we review the evidence for these effects revealed with data from the Rossi Explorer in the last five years. We also discuss the exciting prospects for making the first quantitative tests of strong-gravity general relativistic predictions with a large-area X-ray timing mission.
\end{abstract}

\section{Introduction}

X-ray timing has historically led to fundamental understanding of many phenomena, such as accretion-powered pulsars, low-mass X-ray binaries, and strongly magnetized neutron stars. X-ray timing has unique power because timing signals are often clean diagnostics of systems, and hence lead directly to physical understanding. Here we discuss recent results of X-ray timing. In particular, we focus on aspects of fundamental physics that can be addressed with timing observations. Physics in ultrastrong magnetic fields, high densities, and strong gravity can all be tested with such observations, which reveal truly small-scale structure, from the $\sim 10^{6} \mathrm{~cm}$ sizes of neutron stars and black holes to the $\sim 10^{-13} \mathrm{~cm}$ radii of nucleons. In $\S 2$ we consider strongly magnetized neutron stars, and the evidence for them apparent in timing observations. In $\S 3$ we discuss thermonuclear propagation in X-ray bursts, and what it might tell us about such propagation in other astrophysical settings, such as Type Ia supernovae. In $\S 4$ we examine what has been learned about strong gravity and dense matter through X-ray timing observations. Finally, in $\S 5$ we consider future prospects, and the exciting qualitative advances in these fundamental subjects that are expected from observations with a large-area X-ray timing mission.

\section{Strongly Magnetic Sources}

It has long been recognized (e.g., Erber 1966) that in magnetic fields $B \gtrsim B_{c}=$ $m_{e}^{2} c^{3} / \hbar e=4.414 \times 10^{13} \mathrm{G}$, many otherwise insignificant microphysical processes become important or even dominant. Such processes include single photon pair production, photon splitting, and the existence of a vacuum resonance frequency. Terrestrial laboratories are unable to reproduce the combination of high magnetic field and high photon energy or high electron Lorentz factor necessary to 
test the predictions of quantum electrodynamics in these extreme conditions. The study of high-field neutron stars is therefore important for fundamental physics as well as for the astrophysical understanding it brings.

Observations of soft gamma-ray repeaters provide the best current evidence for supercritical magnetic fields $B>B_{c}$. The first detection of soft gamma-ray repeaters was made in 1979, with the detection of a giant burst on 5 March 1979 from the source SGR 0529-66 in the Large Magellanic Cloud (Mazets et al. 1979) It was only much later that this source and two similar ones (SGR 1900+14 and SGR 1806-20) were recognized as a class potentially distinct from classical gamma-ray bursts. These three sources, a more recently detected fourth member of the class (SGR 1627-41; Woods et al. 1999), and possibly even a fifth (SGR 1801-23; Cline et al. 2000) have long quiescent periods of relatively steady $\sim 10^{34-35} \mathrm{erg} \mathrm{s}^{-1} \mathrm{X}$-ray emission punctuated by episodes of bursting behavior. The bursts are typically short, from $\lesssim 0.1 \mathrm{~s}$ to $\sim 3 \mathrm{~s}$, and have peak estimated isotropic luminosities of $L \sim 10^{39-42} \mathrm{erg} \mathrm{s}^{-1}$. The spectra are quasithermal, with a peak energy at $\sim 20 \mathrm{keV}$.

The first suggestion that soft gamma-ray repeaters might be "magnetars" with supercritical magnetic field strengths was made by Thompson \& Duncan $(1995,1996)$. The cleanest evidence in favor of the magnetar hypothesis has come from timing observations. Modulation of the persistent X-ray emission from SGR 1806-20 was detected during the November 1996 active period (Kouveliotou et al. 1998), at a period of $7.5 \mathrm{~s}$ and with a period derivative $\dot{P}=2.6 \times 10^{-3} \mathrm{~s} \mathrm{yr}^{-1}$. Interpreted using the standard dipole braking model, these values of $P$ and $\dot{P}$ imply a surface dipole field of $\approx 8 \times 10^{14} \mathrm{G}$, nearly twenty times the quantum critical field. Similarly, the $\dot{P}=3.3 \times 10^{-3} \mathrm{~s} \mathrm{yr}^{-1}$ spindown of SGR $1900+14$ (Kouveliotou et al. 1999) plus its $5.2 \mathrm{~s}$ period also implies a surface dipole field of $\approx 8 \times 10^{14} \mathrm{G}$ in this source.

The discovery of the high magnetic field inferred from dipole spindown models of SGR 1806-20 and SGR $1900+14$ is a dramatic confirmation of the magnetar model. Alternative models must of course be considered. For example, Marsden, Rothschild, \& Ligenfelter (1999) have noted that the environments of SGRs are unusually dense, and have suggested that accretion must therefore be reconsidered as the driving mechanism for SGR behavior. However, the nature of the spindown supports strongly the interpretation that SGRs have supercritical magnetic fields, and hence are unique laboratories for the predictions of quantum electrodynamics in ultrastrong magnetic fields.

\section{X-ray Bursts and Thermonuclear Propagation}

Prior to the launch of the Rossi X-ray Timing Explorer (RXTE) it was expected theoretically (e.g., Joss 1978; Fryxell \& Woosley 1982; Nozakura, Ikeuchi, \& Fujimoto 1984; Bildsten 1995) that ignition would happen at some point in the fuel layer and then spread, so that burning would not be uniform over the entire star. These expectations were confirmed by the discovery of brightness oscillations during thermonuclear X-ray bursts from several neutron stars in lowmass X-ray binaries (for reviews see Strohmayer, Zhang, \& Swank 1997; van der Klis 2000). These brightness oscillations are highly coherent $(Q=\nu / \mathrm{FWHM} \sim$ 1000; see, e.g., Strohmayer \& Markwardt 1999) and are extremely stable in 
their frequency from burst to burst in a given source (Strohmayer et al. 1998). Their coherence and stability, plus the recent report of a burst oscillation in the $\mathrm{X}$-ray millisecond pulsar SAX J1808-3658 with a frequency equal to the spin frequency (in 't Zand et al. 2000), establishes that the oscillations occur at the spin frequency or its first overtone. The physical picture is that ignition happens at some spot on the surface, and the burning then propagates away from this spot. At a given moment, there is therefore a hot spot on the surface. Rotational modulation as seen by a distant observer then produces the observed oscillations. The linear rotational velocity, $\sim 0.1 c$, is much greater than the $\sim 10^{6-9} \mathrm{~cm} \mathrm{~s}^{-1}$ velocity expected for thermonuclear propagation, and hence the rotational modulation provides a series of snapshots of the burning as it propagates.

The study of thermonuclear propagation in X-ray bursts has broad implications for other propagation phenomena, such as classical novae and Type Ia supernovae. SNe Ia are especially important to understand because of their unique value as probes of the expansion of the universe and of the possible existence of a cosmological constant. However, these sources are difficult to model numerically, due to the large range of length scales involved (from the $\sim 1 \mathrm{~cm}$ thickness of flame fronts to the $\sim 10^{8} \mathrm{~cm}$ radius of the pre-SN white dwarf). Observations are also challenging, because the propagation of burning happens primarily deep within the white dwarf, and a given source only has one episode of such propagation in its lifetime. This means that, even on such issues as whether the propagation happens as a deflagration (slower than the sound speed $c_{s}$ in the unburned material) or a detonation (faster than $c_{s}$ ), and the expected transition between those propagation regimes, there is considerable uncertainty. Observation of thermonuclear propagation during X-ray bursts offers a different path by which these processes can be understood. The burning happens close to the surface ( $\sim$ meters), where it is relatively easily observed, and happens frequently for a given source, so many observations can be made. Careful measurement of burst brightness oscillations, especially with a future high-area timing instrument, therefore holds promise for the elucidation of thermonuclear propagation in other circumstances such as Type Ia supernovae.

One recent result about propagation emerged from study of the burst source $4 \mathrm{U} 1636-536$, in which the dominant $580 \mathrm{~Hz}$ brightness oscillation is at twice the spin frequency (Miller 1999). This is interpreted as the existence of two nearly antipodal hot spots on the surface, perhaps the result of two pools of fuel (e.g., at the magnetic poles). In general, ignition is expected to happen at one pole first, then propagate to the other, so the rapidity with which the $580 \mathrm{~Hz}$ oscillation appears indicates the speed of propagation. In the case of $4 \mathrm{U} 1636^{-}$ 536 , the $580 \mathrm{~Hz}$ oscillation appears within $0.03 \mathrm{~s}$ of the burst onset. Combined with the $3 \times 10^{6} \mathrm{~cm}$ between antipodes, this implies a propagation velocity $v>10^{8} \mathrm{~cm} \mathrm{~s}^{-1}$, much larger than the $\sim 10^{6} \mathrm{~cm} \mathrm{~s}^{-1}$ expected in some models of turbulent deflagration waves (see, e.g., Fryxell \& Woosley 1982; Nozakura et al. 1984).

These numbers point to one of many qualitative advances that would be possible with an X-ray timing mission with $10 x$ the collecting area of RXTE. The speed of sound in the unburned medium is expected to be $2-3 \times 10^{8} \mathrm{~cm} \mathrm{~s}^{-1}$. With the observed signal strength, an instrument with ten times RXTE's area would 
be able to resolve propagation velocities up to $10^{9} \mathrm{~cm} \mathrm{~s}^{-1}$, and would therefore be able to distinguish between deflagration and a detonation and possibly see a transition between the two burning regimes. This would be a major advance in the understanding of thermonuclear propagation in general.

\section{Strong Gravity and Dense Matter}

Many of the most dramatic advances afforded by X-ray timing have occurred in the last few years, in the subjects of strong gravity and dense matter. This is a direct result of the high area, fast timing, and high telemetry rate of the Rossi X-ray Timing Explorer. In this section we summarize what has been learned from high-frequency brightness oscillations from neutron stars and black holes, and in the next section we discuss the dramatic qualitative advances in our understanding that would be made possible by a future timing mission with ten times the area of RXTE.

\subsection{Neutron stars}

In addition to brightness oscillations during X-ray bursts (discussed in the previous section), the major discovery with RXTE was the existence of $\mathrm{kHz}$ quasiperiodic brightness oscillations (QPOs) in neutron-star low-mass X-ray binary systems and the existence of a number of QPOs in accreting black holes. The QPOs in neutron stars are seen from $\sim 20$ systems, and a number of trends have been established (see van der Klis 2000 for a review). These QPOs (1) often appear as two simultaneous peaks in a power density spectrum, (2) are relatively sharp and strong, with $Q \equiv \nu / F W H M$ up to $\sim 100-200$ in some cases and fractional rms amplitudes up to $15 \%$ in the $2-60 \mathrm{keV}$ range of the Proportional Counter Array on RXTE, (3) tend to increase in frequency as the inferred mass accretion rate increases, (4) have a separation frequency between the two peaks that often decreases with increasing peak frequency, (5) have a maximum separation frequency that is close to the spin frequency inferred from burst brightness oscillations. A number of models have been proposed for this phenomenon (see van der Klis 2000 and references therein). For the reasons discussed in Miller (2000), we favor beat-frequency mechanisms, in which the upper peak frequency is close to the frequency of a circular orbit at some special radius, and the lower peak is generated by a beat of that frequency with radiation modulated at the stellar spin frequency (Miller, Lamb, \& Psaltis 1998). However, many of the most important inferences about strong gravity and dense matter from these oscillations can be derived more generally, from the widely accepted idea that the upper peak frequency $\nu_{2}$ is an orbital frequency.

There are two simple considerations that yield a constraint on the mass $M$ and the radius $R$ of a neutron star, from the observation of an orbital frequency $\nu_{\text {orb. }}$ First, the orbit is obviously outside the star. Second, the orbit must also be outside the innermost stable circular orbit (ISCO), because otherwise there would be rapid inspiral and any resulting oscillation would last at most a few cycles, leading to a broad QPO, in conflict with observations. As shown by Miller et al. (1998), these limits constrain the mass of the star to be $M<$ $2.2(1+0.75 j)\left(1000 \mathrm{~Hz} / \nu_{2}\right) M_{\odot}$ and the circumferential radius to be $R<19.5(1+$ $0.2 j)\left(1000 \mathrm{~Hz} / \nu_{2}\right) \mathrm{km}$, to first order in the dimensionless spin parameter $j \equiv$ 
$c J / G M^{2}$, where $J$ is the stellar angular momentum $(j \approx 0.1$ for a neutron star with a spin frequency of $300 \mathrm{~Hz}$, and the spacetime is unique to first order in $j$ ). Together, the observation of a given orbital frequency restricts the mass and radius to be inside a wedge in a $M-R$ diagram; the larger the frequency, the more restrictive the constraint. The highest frequency yet observed, $1330 \mathrm{~Hz}$ (van Straaten et al. 2000), rules out the hard equation of state L (labeled as in Miller et al. 1998). This is the first time that an astrophysical observation has been able to rule out a hard equation of state. This is therefore a major advance in our understanding of the dense matter in the cores of neutron stars.

As first discussed in 1996, an even more major advance would occur if strong evidence were found for the existence of the ISCO. This is because the existence of unstable circular orbits is a qualitatively new feature of general relativity compared to Newtonian gravity, and is an essential component of models of accreting black holes on all scales as well as of inspiraling compact objects and other phenomena. Given that observed frequencies increase with increasing $\dot{M}$, but that $\nu_{\mathrm{ISCO}}$ is an upper limit to the frequency of the upper peak, it was expected that there would be a rollover in the slope of the $\nu-\dot{M}$ curve for both the upper and lower peaks (Miller et al. 1998). Moreover, the frequency at which the curve rolled over has to be a constant, for many observations of a given source. This may have been seen in RXTE data from 4 U 1820-30 by Zhang et al. (1998). If so, this is a tremendous discovery; not only is this strong confirmation of the prediction of the ISCO, but it implies a neutron star gravitational mass of $2.15 M_{\odot}$, which limits the possible high-density equation of state severely. Given these dramatic implications, care must be taken in the interpretation of the data. The main question is whether the rollover really happens as a function of $\dot{M}$, or whether the countrate change is indicative of a spectral state change. Further observations and additional sources with this signature would help immensely. This is one of the many potential benefits of a future large-area timing instrument.

\subsection{Black holes}

There are many different types of quasiperiodic oscillations from black hole sources discovered with RXTE. One that has been seen in the microquasars GRS $1915+105$ and GRO J1655-40 is particularly intriguing. The frequency appears not to change with luminosity in either source (fixed at $67 \mathrm{~Hz}$ for GRS $1915+105$ and at $\approx 300 \mathrm{~Hz}$ for GRO J1655-40). In GRS $1915+105$ the feature can be fairly sharp, with a maximum $Q \approx 20$. These QPOs are much weaker than many QPOs seen in neutron star sources, with typical rms amplitudes $\sim 1 \%$. They are therefore more difficult to study, and less is known about them. However, their fixed frequency suggests that they are related to some fundamental quantity or radius in the system; possibilities include frame-dragging frequencies due to Lense-Thirring precession or periastron advance (e.g., Stella, Vietri, \& Morsink 1999), and disk oscillation modes (e.g., Nowak et al. 1997). Regardless of the physical origin of these QPOs, the matter generating the oscillations is close enough to the black hole that it must in some sense reflect the strongly curved spacetime. It is therefore expected that, especially with the well-characterized waveforms available with a large-area timing mission, the 
mass and spin of the black hole could be inferred, and potentially the spacetime itself might be probed.

\section{Future Prospects}

An X-ray timing satellite with ten times the collecting area of RXTE would be the first instrument able to characterize accurately the waveforms and energydependent phase lags of the high-frequency QPOs observed from compact objects in low-mass X-ray binaries. As described below, these waveforms and phase lags encode otherwise inaccessible information about strong gravity and the properties of the compact objects. With this capability, such an instrument would test quantitatively the predictions of general relativity in the highly curved spacetime near neutron stars and black holes. It would also make fundamentally new contributions to our understanding of the properties of matter at supranuclear densities.

\subsection{Waveforms and Phase Lags}

The waveform from a rotating hot spot during a burst oscillation or persistent emission is affected by gravitational light deflection and Doppler shifts. By fitting the waveform one can therefore constrain simultaneously the mass and radius of the star, and hence the equation of state of matter beyond nuclear saturation density, which is an issue of central importance in nuclear physics research. Fig. 1a shows that observations from a large-area timing instrument would bring an unprecedented level of precision to our understanding of highdensity matter. In this simulation we assume a neutron star of gravitational mass 1.8 $M_{\odot}$ with high-density equation of state UU, and assume that the hot spot is a small spot on the rotational equator, as seen by a distant observer in the rotational plane. We simulate five bursts which had $5 \% \mathrm{rms}$ oscillations at $364 \mathrm{~Hz}$ for five seconds each. Modern equations of state have mass-radius curves that span approximately the range between the FPS and UU equations of state (Akmal, Pandharipande, \& Ravenhall 1998). It is clear that measurements of this type for several different neutron stars would exclude most of these EOS. These results would therefore have direct feedback on nuclear physics. Similarly, accurate measurement of the waveforms of luminous clumps around a black hole would yield a precise mass for the hole and strong constraints on the Kerr spin parameter $j=a / M$. It may even be possible to detect the frequency chirp produced as a clump moves inside the last stable orbit (Sunyaev 1973, Stoeger 1980).

The high velocity of surface rotation during bursts or persistent coherent emission also Doppler-boosts the energies of photons from the hot spot, causing high-energy photons to lead low-energy photons by a phase that depends on the precise value of the surface velocity and therefore on the stellar radius, for fixed frequency. This effect was first pointed out by Ford (1999) in an analysis of RXTE data on bursts from Aql X-1, and has also been analyzed for the coherent oscillations from the millisecond X-ray pulsar SAX J1808-3658 by Weinberg, Miller, \& Lamb (2000) and Ford (2000). In Fig. 1b we simulate a fit to such phase lag data, with an assumed gravitational mass of $2.0 M_{\odot}$ and equation of state UU, and phase uncertainties taken from the SAX J1808-3658 data. This 

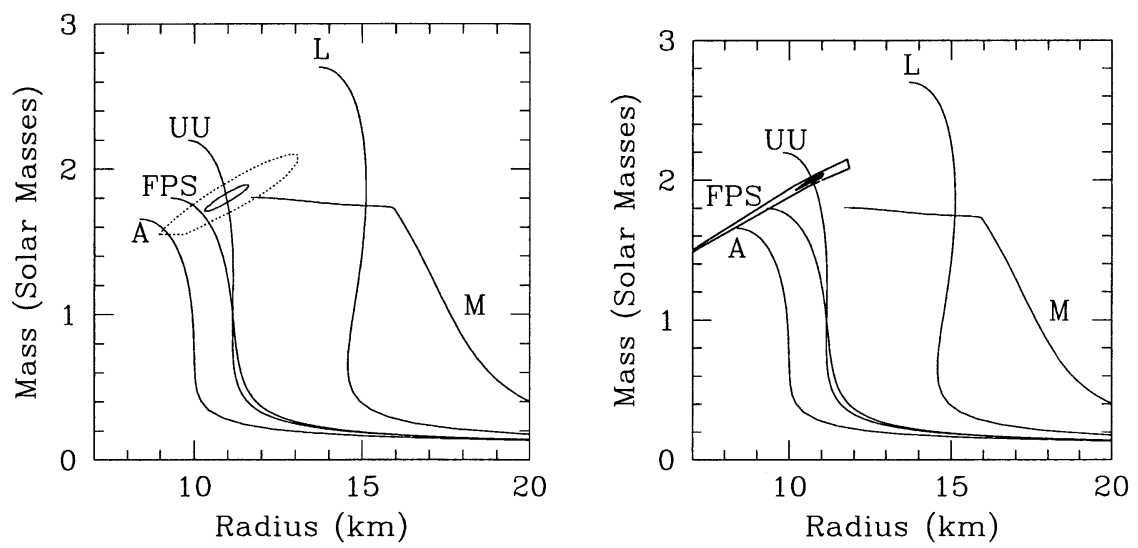

Figure 1. (left panel) Constraints on mass and radius possible with waveform fitting of burst brightness oscillations. We assume the UU high-density equation of state and a gravitational mass of $1.8 M_{\odot}$. See text for other details of the simulations. The dotted outer contour and solid inner contour show, respectively, the $1 \sigma$ confidence region possible with RXTE and with a future instrument with ten times the area of RXTE. The light solid curves show the mass-radius relations given by different high-density equations of state, labeled as in Miller, Lamb, \& Psaltis (1998). (right panel) Constraints on mass and radius from phase lag fitting of coherent oscillations from a millisecond pulsar. Phase uncertainties were adopted from measurements of SAX J18083658 , and we assumed the UU high-density equation of state and a gravitational mass of $2.0 M_{\odot}$ (indicated by the black dot). The outer and inner contours show the $1 \sigma$ uncertainty region possible with, respectively, RXTE and a future instrument with ten times the area of RXTE.

figure shows that although RXTE is not sufficiently sensitive to provide useful constraints, an observation with a future large-area timing instrument would yield tight limits on the mass and the radius of this neutron star, and hence on the equation of state of high-density matter.

\subsection{Quantitative tests of general relativity}

The most exciting prospect of a high-area timing mission is that data from it could test quantitatively the predictions of general relativity in strong gravity. In the previous sections we have discussed several independent methods of estimating the mass, radius, or angular momentum of an individual neutron star or black hole. These include waveform and phase lag fits and the detection of an ISCO and the associated orbital frequency. The combination of all these independent measurements overdetermines the problem so that the underlying theories get tested. For example, the various estimates of the mass of the central object will agree with each other only if general relativity is the correct theory of gravity, because general relativity is assumed in making the estimates. This 
would provide a powerful test of its predictions in a regime that is otherwise not accessible, and would continue the legacy of X-ray timing as a clean measuring tool for fundamental physics and astrophysics.

Acknowledgments. This work was supported in part by NASA grant NAG 5-9756.

\section{References}

Akmal, A., Pandharipande, V. R., \& Ravenhall, D. G. 1998, Phys. Rev. C58, 1804

Bildsten, L. 1995, ApJ, 438, 852

Cline, T., et al. 2000, ApJ, 531, 407

Erber, T. 1966, Rev. Mod. Phys., 38, 626

Ford, E. C. 1999, ApJ, 519, L73

Ford, E. C. 2000, ApJ, 535, L119

Fryxell, B. A., \& Woosley, S. E. 1982, ApJ, 258, 773

in 't Zand, J. et al. 2000, A\&A, submitted

Joss, P. C. 1978, ApJ, 225, L123

Kouveliotou, C. et al. 1998, Nature, 393, 235

Kouveliotou, C. et al. 1999, ApJ, 510, L115

Marsden, D., Rothschild, R. E., \& Ligenfelter, R. E. 1999, ApJ, 523, L97

Mazets, E. P., et al. 1979, Nature, 282, 587

Miller, M. C. 1999, ApJ, 515, L77

Miller, M. C. 2000, Astr. Lett. Comm., in press (astro-ph/0007287)

Miller, M. C., Lamb, F. K., \& Psaltis, D. 1998, ApJ, 508, 791

Nowak, M. A., et al. 1997, ApJ, 477, L91

Nozakura, T., Ikeuchi, S., \& Fujimoto, M. Y. 1984, ApJ, 286, 221

Stella, L., Vietri, M., \& Morsink, S. M. 1999, ApJ, 524, L63

Stoeger, W. R. 1980, MNRAS, 190, 715

Strohmayer, T. E., \& Markwardt, C. B. 1999, ApJ, 516, L81

Strohmayer, T. E., Zhang, W., \& Swank, J. H. 1997, ApJ, 487, L77

Strohmayer, T. E., et al. 1998, ApJ, 503, L147

Sunyaev, R. A. 1973, Sov. Astr. AJ., 16, 941

Thompson, C., \& Duncan, R. C. 1995, MNRAS, 275, 255

Thompson, C., \& Duncan, R. C. 1996, ApJ, 473, 322

van der Klis, M. 2000, ARA\&A, 38, 717

van Straaten, S., et al. 2000, ApJ, 540, 1049

Weinberg, N., Miller, M. C., \& Lamb, D. Q. 2000, ApJ, in press

Woods, P. M. et al. 1999, ApJ, 519, L139

Zhang, W., et al. 1998, ApJ, 500, L171 